\title{
PARTICLE SWARM-AIDED DESIGN OF A FUZZY LOGIC STABILIZER FOR A SUPERCONDUCTING GENERATOR IN A MULTI-MACHINE POWER SYSTEM
}

\author{
Ragaey A. F. Saleh \\ Department of Electrical Engineering; Faculty of Engineering, \\ Minoufiya University, Shebin El-Kom, Egypt
}

\begin{abstract}
The application of a fuzzy logic stabilizer to enhance the stability of a superconducting generator (SCG) in a multi-machine power system is described. The proposed fuzzy stabilizer uses SCG speed deviation and acceleration as input signals; The stabilizer parameters set includes the scaling factors for input and output variables, as well as the overlap ratios of neighboring fuzzy membership functions of input variables. Particle swarm optimization (PSO) technique is employed to search for optimal settings of the fuzzy stabilizer parameters. Simulation results show that the proposed; PSO-tuned fuzzy stabilizer provides good damping to SCG in a multi-machine environment when operating in conjunction with conventional stabilizers on other machines.

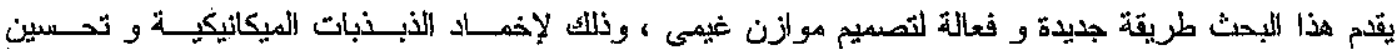

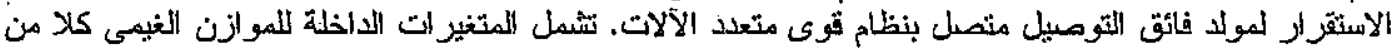

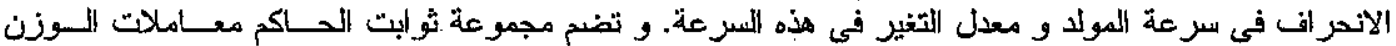

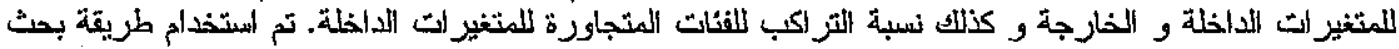

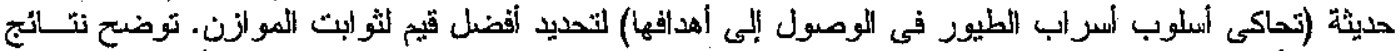

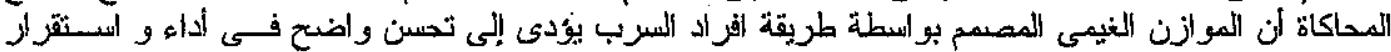
المنظام المدروس المبان.
\end{abstract}

Keywords: Particle swarm optimization, Fuzzy logic stabilizer, Superconducting generator, Multimachine system

\section{INTRODUCTION}

The application of superconductors to electric power apparatus is considered a key technology for the current century. The electric power demand has been steadily increased. worldwide. This tendency will continue in the future, and therefore the capacities of the power transmission systems have to increase. Large power systems require developing a more efficient and stabilizing technology for large amounts of power transmission. One promising method is to introduce the superconducting generator (SCG), which has a very low synchronous reactance [1]. Superconducting generators have also many other potential advantages compared with the conventional generators such as higher efficiency and smaller size and weight. The attractions of SCG have drawn more interest in industrial countries since 1970's, especially in Japan where many R\&D projects on SCGs have been conducted at utility companies, power plant manufacturers and other organization toward 200 MW class pilot machine [2-3]. Despite these advantages, SCG field winding has an extremely. large time constant. The excitation system is therefore not able to change quickly the field current to meet the grid requirements under transient conditions. Inevitably, the only - control means feasible to enhance SCG stability following power system faults is the fast acting governors on the steam supplies to the turbine.

Transient stability is one of the most important issues that should be investigated in power system planning, operation, and expansion. It is mainly concerned with maintaining generator synchronization following a sudden and major disturbance or an abrupt change in load or generation power. The importance of this issue increases when considering a superconducting generator in a multi-machine system. In the past, a number of investigations have been conducted to study and improve the behaviour of a superconducting generator in a multi-machine system [4-5], The results reported in [4] show that the incorporation of a SCG in a multi-machine system increases its stability reserve, but slightly reduces the overall damping of the system. However, a good improvement in the performance and stability limits can be achieved by using a conventional lead stabilizer in the governor loop of the SCG [5]. Alternative stabilizers based on adaptive control techniques have been proposed [6-8]. However, the on-line parameter identification is still questionable especially during fault periods. Recently, fuzzy logic control has emerged as one of the most fruitful 
research areas, and many applications for enhancing power system stability have been reported in literature [9-10]. The fuzzy logic stabilizer is essentially a multi-parameter controller, whose performance depends on the shape of membership functions, rule base and scaling factors. However, the design of a fuzzy stabilizer with satisfactory performance is a rather difficult problem. To overcome this problem, genetic algorithm (GA) was proposed as an efficient technique for the optimal design of power system stabilizers [11-12]. More recently, a new heuristic search method called particle swarm optimization (PSO) has been introduced [13-14]. PSO is characterized as a simple concept, easy to implement, and computationally efficient. Theses features make PSO technique able to accomplish the same goal as GA optimization in a new and faster. way. A number of very recent successful applications of PSO on various power system problems have been reported in literature [1517]. The objective of this paper is to enhance the stability of a SCG in a multi-machine system using fuzzy governor controller optimally designed by the PSO technique.

\section{SYSTEM UNDER STUDY}

The multi-machine system under consideration is shown in Fig. 1. It is a twelve-bus four-machine power system. The machine at bus 3 is a superconducting generator, while the other three machines are conventional generators. The four generating units are connected to four load areas as shown in the figure. Based on Park's d-q axis representation, each conventional machine is modeled by seven non-linear differential equations. The order of SCG model is increased to nine to accommodate. the double-screened rotor. Transmission lines are modeled using the $\pi$-method, and the loads are represented by constant impedances. Each conventional generator is equipped with a typical excitation system and a conventional power system stabilizer (PSS) having the transfer function $G_{s}(1+0.15 s) /(1+0.015 s)$ [18]. The block diagram of the excitation system is shown in Fig. 2. In this study, the mechanical input to each conventional generator is assumed constant. Meanwhile, a detailed representation for the prime mover of the SCG is used, because it is the main concern of this study. The SCG is driven by a threestage steam turbine with reheat. The turbine is controlled by fast acting electro-hydraulic governors fitted to the main and interceptor valves, which are working in unison. Mathematical models for $\mathrm{SCG}$, turbine and governors, along with the system parameters are given in the Appendix.

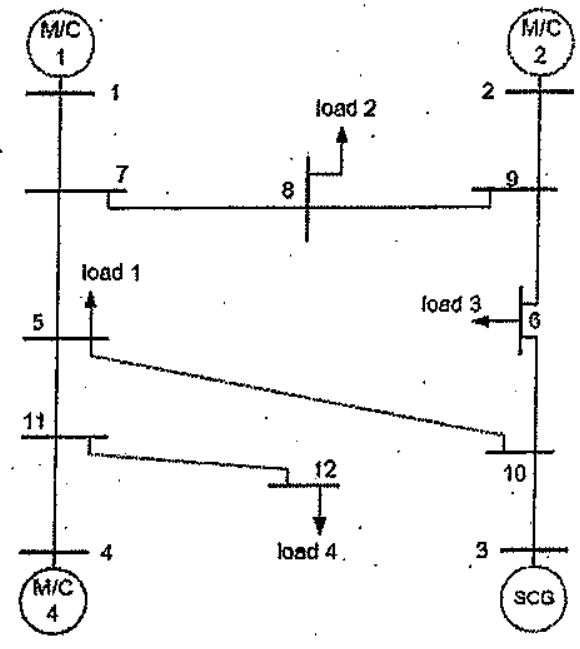

Fig.1 Four-machine power system

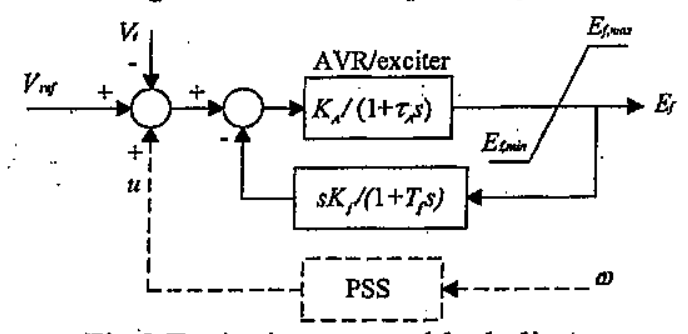

Fig.2 Excitation system block diagram

\section{3- PARTICLE SWARM OPTIMIZATION}

Particle swarm optimization (PSO) technique is an efficient, global search technique recently introduced by Kennedy and Eberhart [13]. PSO models the behavior and cooperation aspects of individual members within a social system such as flock of birds or school of fishes. In this model, the system is populated with individual particles, referred to as "swarm", representing possible solutions to the problem considered. Particles fly around in a multidimensional search space. During flight, each particle adjusts its position according to its own experience, and experience of neighboring particles, making use of the best position encountered by itself and its neighbors. The algorithm of PSO technique is much simpler than that of GA. In PSO algorithm, each solution can be represented as a particle in a swarm, having a position and velocity. Each position coordinate represents a parameter value. Thus, for an n-dimensional optimization, each particle has a position in n-dimensional space that represents a solution [14]. The PSO starts with generation of initial swarm particles, assigning a random position and a random velocity for each particle. Ther, PSO algorithm evaluates each particle's fitness using a predefined fitness (objective) function. The position with the highest fitness value in the entire run is referred to as "global best position" ( $g_{\text {best }}$ ). Meanwhile, each particle keeps track of its highest 
fitness value. The location of this value is called "personal best position" $\left(p_{\text {best }}\right)$. The algorithm then proceeds by updating the velocity of each particle using its current velocity and its distance from $g_{b e s t}$ and $p_{b e s t}$ according to the following equation:

$$
\begin{aligned}
& v_{i}^{k}=w^{k} v_{i}^{k-1}+c_{1} r_{i}\left(p_{\text {bess }, i}-x_{i}^{k-1}\right) \\
& +c_{2} r_{2}\left(g_{\text {best }, i}-x_{i}^{k-1}\right) \\
& i=1,2,3, \ldots \ldots \ldots \ldots \ldots \\
& v_{i}^{k} \quad \text { is the velocity of particle } i \text { at iteration } k \\
& x_{i}^{k} \quad \text { is the position of particle } i \text { at iteration } k \\
& r_{1}, r_{2} \text { are uniformly distributed random numbers in } \\
& \text { the range }[0,1] \\
& c_{1}, c_{2} \text { are positive constants } \\
& w^{k} \quad \text { is the inertia weight at iteration } k \\
& n \text { is the number of particles in a swarm }
\end{aligned}
$$

The inertia weight is one of the main elements of PSO technique. It is used to control the impact of the previous velocity on the current velocity. Proper. choice of inertia weight provides a balance between global and local exploration abilities of the particles. As originally developed, large inertia weight is recommended at initial stages of the search process to enhance the global exploration, while lower values of the inertia weight are preferred at final stages to improve local exploration. The inertia weight can be decreased linearly over search iterations from a maximum value (usually 0.9 ) to a minimum value (usually 0.4 ). Alternatively, the inertia weight may be decreased in a non-linear form as follows [15]:

$$
w^{k}=\alpha w^{k-1}
$$

Where $\alpha$ is a decrement constant smaller than but close to l. Another important parameter of PSO procedure is the maximum velocity $\left(V_{m a x}\right)$ of a particle in any given dimension. This parameter determines the resolution with which the search space is explored. If $V_{\max }$ is too high, a particle might fly past good solutions. Meanwhile, if $V_{\max }$ is too small, a particle may be trapped in a local solution. The maximum velocity is often set at $10-20 \%$ of the range of the variable in each dimension. Therefore, the magnitude of particle velocity is always limited by $V_{\max }$. After updating the velocities, the position of each particle is modified according to the following equation:

$x_{i}^{k}=x_{i}^{k-1}+v_{i}^{k}$

The algorithm proceeds by updating the best position of each particle according to its new position; the global best position is then updated as well. This procedure is repeated until a specified termination condition is met.

\section{FUZZY LOGIC STABMIZER}

In this section, the determination of an efficient control signal, $u$, based on fuzzy logic is described. This signal is then introduced into the governor side of the SCG turbine as shown in Fig. 3.

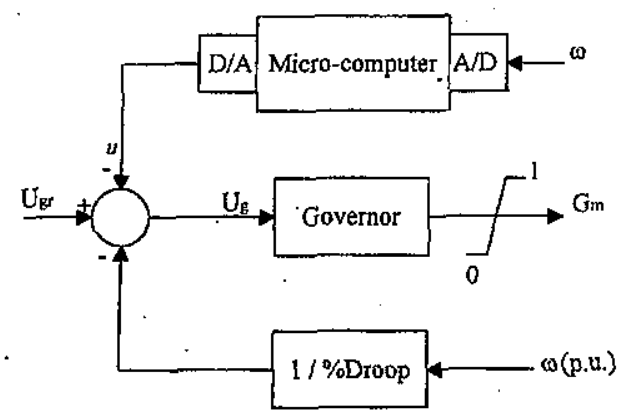

Fig.3 The governor control system

Speed deviation, $\omega$, and its derivative, $\dot{\omega}$, are chosen as input variables. Actually only $\omega$ signal is measured, and from it $\dot{\omega}$ signal is computed as:

$\dot{\omega}(k)=[\omega(k)-\omega(k-1)] / T_{s}$

where $T_{s}$ is the sampling interval. Two scaling factors, $K_{A}$ and $K_{B}$, are used to map $\omega$ and $\dot{\omega}$, respectively into their predefined universes of discourse, which are divided into seven overlapping fuzzy sets; named large positive "LP", medium positive "MP", small positive "SP", zero "ZE", small negative "SN', etc. A triangle membership function is assigned for each fuzzy set such that if a crisp input " $x$ " belongs to a set of range [a-b], width " $d$ " and center " $c$ ", then its degree of membership $\mu_{x}$, in this set is defined by the following function:

$$
\mu_{x}=\left\{\begin{array}{lc}
(2(x-a) / d) & \text { if } \mathrm{a} \leq \mathrm{x} \leq c \\
(2(b-x) / d) & \text { if } \mathrm{c} \leq \mathrm{x} \leq b \\
0 & \text { else }
\end{array}\right.
$$

Table 1 shows the fuzzy rules that are assigned for the SCG system. Each entry in Table 1 represents a control rule, which takes the form: "IF $\omega$ is A, AND $\dot{\omega}$ is $\mathrm{B}$, THEN u is C", where $A, B$, and C are fuzzy sets as defined by relation (5). These fuzzy rules are individually applied on the fuzzified inputs, resulting in an output fuzzy set, for each rule, clipped to a degree defined as:

$$
\mu_{c}\left(u_{i}\right)=\min \left(\mu_{A}(\omega), \mu_{B}(\dot{\omega})\right)
$$

The aggregated fuzzy outputs are converted into a single crisp value using the "weighted average" defuzzification method, which gives the output control signal as: 


$$
u=K_{u} \frac{\sum_{i=1}^{m} \mu_{c}\left(u_{i}\right) \cdot u_{i}}{\sum_{i=1}^{m} \mu_{c}\left(u_{i}\right)}
$$

where $K_{u}$ is a scaling factor, $m$ is the number of rules giving contribution to the fuzzy output at the sampling instant considered, and $u_{i}$ is the center value of the fuzzy set in consequent $i$. According to the structure of fuzzy logic stabilizer described above, the number of fuzzy sets, to which an input value belongs at a time; depends on how much overlap between adjacent fuzzy sets is.

Table 1: Fuzzy logic control rules for SCG system

\begin{tabular}{|c|c|c|c|c|c|c|c|}
\hline datd & NL & NM & NS & ZE & PS & PM & PL \\
\hline NL & NS & PS & PM & PM & PM & PL & PL \\
\hline NM & NS & NS & PS & PS & PM & PM & PL \\
\hline NS & NM & NS & NS & PS & PS & PM & PM \\
\hline ZE & NM & NM & NS & ZE & PS & PM & PM \\
\hline PS & NM & NM & NS & NS & PS & PM & PM \\
\hline PM & NL & NM & NS & NS & PS & PS & PS \\
\hline $\mathbf{P L}$ & NL & NL & NM & NM & NS & NS & PS \\
\hline
\end{tabular}

\section{PSO-BASED STABILIZER PARAMETERS SELECTION}

The tuning parameters of the fuzzy stabilizer are $K_{A}$, $K_{B}$ and $K_{k}$. To increase the effectiveness of the proposed stabilizer, additional two other adjustable parameters are introduced into the design. Namely, $R_{A}$ which stands for the $\mathrm{x}$-axis overlap ratio between adjacent fuzzy sets of $\omega$, and $R_{B}$ which stands for the $x$-axis overlap ratio between adjacent fuzzy sets of $\dot{\omega}$. Therefore, we have now five parameters $\left(K_{A}, K_{B}, K_{u}, R_{A}, R_{B}\right)$ to be optimally chosen. This task is achieved using the PSO technique. First, a quadratic performance index is defined as:

$$
J=\left(\sum_{k=1}^{N}[\omega(k)]^{2}\right)^{0.5}
$$

where $a(k)$ is the deviations of the SCG speed from the steady state value. The problem of designing a fuzzy logic stabilizer is then transformed into an optimization problem, where PSO is utilized off-line to select the stabilizer parameters. The proposed stabilizer was designed at the loads and operating points of case \#1 shown in Table 5. However, like many recursive and stochastic methods, PSO itself has a number of parameters to be properly specified. The main PSO parameters are the initial inertia weight, $w^{0}$, and the maximum allowable velocity, $V_{\text {max }}$. The initial inertia weight is set at 1 , and $V_{\max }$ at $12.5 \%$ of the search space of each variable. The swarm size of PSO is chosen to be 60 particles. Other parameters are set as decrement constant $\alpha=0.98$, and $c_{1}=c_{2}=2$.

\section{SIMULATION RESULTS}

In this study, the SCG exciter voltage was kept constant during transients. The optimization process was carried out in response to a three-phase to ground fault of $200-\mathrm{ms}$ duration at bus 5 at the end of line 5 10. Variation of the performance index $J$ with the number of iterations is shown in Fig. '4. The optimal stabilizer parameters selected by PSO are $\mathrm{K}_{\mathrm{A}}$ $=0.75, K_{B}=0.328, K_{u}=2, R_{A}=0$ and $R_{B}=0.478$.

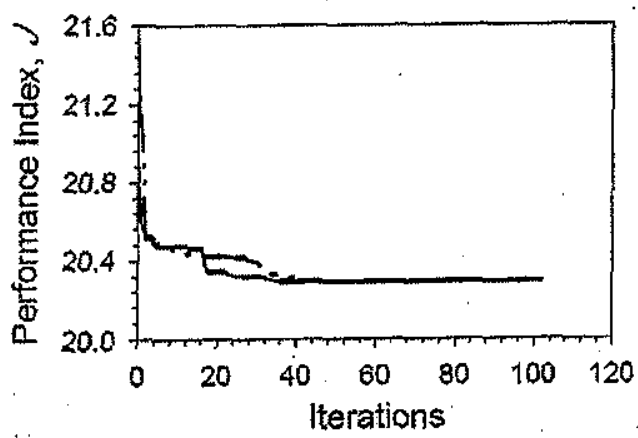

Fig.4 Convergence of performance index with different initializations

Since there is no infinite-bus, machine 4 was taken as a reference unit. The rotor angles of the other machines are shown .with respect to that of the reference unit. The multi-machine system performance was obtained at three situations. First, when the four generators are not equipped with stabilizers. Second, when each conventional generator is equipped with a conventional PSS, while the SCG is stabilized via a governor lead stabilizer [5]. Third, as in second, but the governor lead stabilizer is replaced with the governor fuzzy stabilizer designed above. The SCG performance is shown in Fig. 5, Fig 6, and Fig. 7. These figures also show the performance of other machines in the system. Fig. 8 shows the system response to the same fault, but with loads and operating points given under case \#2 in Table 5.

The simulation results show that the incorporation of the PSO-based fuzzy stabilizer in the governor loop of the SCG leads to a significant improvement in the SCG performance and an appreciable increase in damping of the rotor oscillations with a reduction in the rotor first swing. This can clearly be noticed from Fig. 5(a), Fig. 5(c) and Fig. 6. In addition, the results show that the SCG performance with the proposed fuzzy stabilizer is almost similar to the performance with the conventional lead stabilizer. However, more degrees of freedom could be added to the design of the fuzzy stabilizer to enhance its effectiveness. The authors are now working on this point. 


\section{CONCLUSTON}

This paper has presented an approach for the design of a fuzzy logic stabilizer for transient performance improvement of a SCG operating in a multi-machine system. A set of fuzzy decision rules relating the SCG status, in terms of its speed deviation and acceleration, to the control action required was assigned based on previous experience with controller design. A performance index was defined, and then PSO technique was used to optimize a set of unknown stabilizer parameters at the specified loads. The results of non-linear simulation study show the effectiveness of the proposed PSO-tuned fuzzy stabilizer in damping the rotor oscillations and therefore enhancing the SCG stability.

\section{8- REFERENCES}

[1] T. Nitta, et. al., "Transient stability limit issues at three-phase short-circuit in parallel running of both a superconducting generator and a conventional one", Electrical Engineering in Japan, Vol. 115, No. 6, pp. 62-70, 1995.

[2] K. Ueda, et. al., "Measurement and analysis of 70 MW superconducting generator constants", IEEE Trans. on Applied Superconductivity, Vol.9, No.2, pp.1193-1196, 1999.

[3] H. Tsukiji, T. Hoshino, and I. Muta, "Output power limit of $200 \mathrm{MW}$ class brushless superconducting generator excited with magnetic flux pump" IEEE Trans. on Applied Superconductivity, Vol.11, No.1, pp.2335-2338, 2001.

[4] Y.H.A. Rahim, et. al., "Stability of a multimachine system incorporating a superconducting generator", IEEE trans. on EC, Vol. 3, No.3, pp.458-464, 1988.

[5] S.M. Osheba, et. al., "Comparison, of transient performance of superconducting and conventional generators in a multi-machine system", IEE Proc., Pt. C, Vol. 135, No.5, pp. 389-395, 1988.

[6] Q.H. Wu, and B.W. Hogg, "Adaptive controller for a turbogenerator system", IEE Proc. D, Vol.135, pp. 35-42, 1988.

[7] C.M. Lim and T. Hiyama, "Self tuning control scheme for stability enhancement of multimachine power systems", IEE Proc., Vol.137, Pt.C, No.4, pp. 269-275, 1990.

[8] G.P. Chen, O.P. Malik, G.S. Hope, Y.H. Qin and G.Y. Xu, "An adaptive power system stabilizer based on the self optimizing pole shifting control strategy", IEEE Trans. on EC, Vol.8, No.4, pp.639-645, 1993.
[9] T. Hiyama, "Robustness of fuzzy logic power system stabilizers applied to multimachine power system", IEEE Trans. EC, Vol.9, No.3, pp. 451-459, 1994.

[10] K.A. El-Metwally, G.C. Hancock, and O.P. Malik, "Implementation of a Fuzzy logic PSS using a micro-controller and experimental test results", IEEE Trans. EC, Vol.11, No.1, pp.9196, 1996.

[11] J. Wen, et. al., "A synchronous generator fuzzy excitation controller optimally designed with a genetic algorithm", IEEE Trans. on Power Systems, Vol.13, No.3, pp. 884-889, 1998.

[12] R.A.F. Saleh, and H.R. Bolton, "Genetic algorithm-aided design of a fuzzy logic stabilizer for a superconducting generator", IEEE Trans. on Power Systems, Vol.15, No.4, pp.1329-1335, 2000.

[13] J. Kennedy and R. Eberhart, "Particle swarm. optimization", Proc. of IEEE Int. Conf. on Neural Networks, Vol. IV, pp.1942-1948, 1995.

[14] R. Eberhart and Y. Shi., "Particle swarm optimization: developments, applications and resources", Proc. of the 2001 Congress on Evolutionary Computation, Vol. 1, pp.81-86, 2001.

[15] M.A. Abido, "Optimal design of power system stabilizers using particle swarm optimization", IEEE Trans. on EC, Vol.17, No.3, pp.406-413, 2002.

[16] J.B. Park, et. al., "A particle swarm optimization for economic dispatch with non-smooth cost functions", IEEE Trans. on Power Systems, Vol.20, No.1, pp.34-41, 2005.

[17] A.A. Abou El-Ela, et. al., "Power systems operation using particle swarm optimization technique", Electric Power Systems Research 78, pp. 1906-1913, 2008.

[18] P.M. Anderson, and A.A. Fouad, "Power system control and stability ", IEEE Press, New York, 2nd edition, 1994.

\section{APPENDIX}

The mathematical model of SCG:

$$
\begin{aligned}
& p \psi_{f}=\omega_{o}\left[V_{f}-i_{f} R_{f}\right] \\
& p \psi_{d}=\omega_{o}\left[V_{d}+i_{d} R_{a}+\psi_{q}\right]+\psi_{q} \omega \\
& p \psi_{D 1}=-\omega_{o} i_{D 1} R_{D 1} \\
& p \psi_{D 2}=-\omega_{o} i_{D 2} R_{D 2} \\
& p \psi_{q}=\omega_{o}\left[V_{q}+i_{q} R_{a}-\psi_{d}\right]-\psi_{d} \omega
\end{aligned}
$$


Ragaey A. F. Saleh, "Particle Swarm-Aided Design of a Fuzzy Logic Stabilizer for a Superconducting...."

$$
\begin{aligned}
& p \psi_{Q 1}=-\omega_{o} i_{Q 1} R_{Q 1} \\
& p \psi_{Q 2}=-\omega_{o} i_{Q 2} R_{Q 2} \\
& p \delta=\omega \\
& p \omega=\frac{\omega_{o}}{2 H}\left[T_{m}-T_{e}\right] \\
& T_{e}=\psi_{d} i_{q}-\psi_{q} i_{d}
\end{aligned}
$$

The mathematical model of the turbine and governor system:

$$
\begin{aligned}
& p Y_{H P}=\left(G_{M} P_{o}-Y_{H P}\right) / \tau_{H P} \\
& p Y_{R H}=\left(Y_{H P}-Y_{R H}\right) / \tau_{R H} \\
& p Y_{I P}=\left(G_{I} Y_{R H}-Y_{I P}\right) / \tau_{I P} \\
& p Y_{L P}=\left(Y_{I P}-Y_{L P}\right) / \tau_{L P} \\
& T_{m}=F_{H P} Y_{H P}+F_{I P} Y_{I P}+F_{L P} Y_{L P} \\
& p G_{M}=\left(U_{g}-G_{M}\right) / \tau_{G M} \\
& p G_{I}=\left(U_{g}-G_{I}\right) / \tau_{G I}
\end{aligned}
$$

Parameters of SCG, turbine and governor systems (inductance and resistance values in p.u; time constants in seconds)

$L_{f}=0.541, \quad L_{d}=L_{q}=0.5435, \quad L_{D I}=L_{Q 1}=0.2567$, $L_{D 2}=L_{Q 2}=0.4225, L_{f d}=L_{f D I}=L_{d D I}=L_{d D 2}=L_{D I D 2}=0.237$, $L_{f D 2}=0.3898, \quad L_{q Q 1}=L_{q Q 2}=L_{Q 1 Q 2}=0.237, \quad \tau_{\mathrm{f}}=750$, $R_{d}=R_{q}=0.003, R_{D I}=R_{Q P}=0.01008, R_{D 2}=R_{Q 2}=0.00134$, $H=3 \mathrm{~s}, \tau_{G M}=\tau_{G I}=0.1, \tau_{H P}=0.1, \tau_{R H}=10, \tau_{I P}=\tau_{L P}=0.3$, $P_{o}=1.2$ p.u. $, F_{H P}=0.26, F_{I P}=0.42, F_{L P}=0.32$

Table 2: Parameters of conventional generators

\begin{tabular}{|ll|l|l|l|}
\hline \multicolumn{2}{|l|}{$\begin{array}{l}\text { Parameter } \\
\text { symbol }\end{array}$} & M/C 1 & M/C 2 & M/C 4 \\
\hline$L_{d}$ & (p.u) & 2.11 & 2.13 & 0.898 \\
$L_{q}$ (p.u) & 2.02 & 2.07 & 0.646 \\
\multicolumn{2}{|l|}{$M_{d F}=M_{d D}=$} & & & \\
$M_{F D}$ & (p.u) & 1.955 & 1.88 & 0.658 \\
$M_{q Q}$ & (p.u) & 1.865 & 1.82 & 0.406 \\
$L_{F}$ & (p.u) & 2.089 & 2.12 & 0.724 \\
$L_{D}$ & (p.u) & 2.07 & 1.97 & 0.668 \\
$L_{Q}$ & (p.u) & 1.93 & 1.88 & 0.457 \\
$R_{d}$ & (p.u) & 0.0046 & 0.0029 & 0.0014 \\
$R_{F}$ & (p.u) & 0.00013 & 0.00092 & 0.00026 \\
$R_{D}$ & (p.u) & 0.02 & 0.018 & 0.012 \\
$R_{Q}$ & (p.u) & 0.024 & 0.0212 & 0.02 \\
$H$ & (s) & 2.32 & 2.52 & 5.15 \\
\hline
\end{tabular}

Table 3: Parameters of excitation systems and PSS

\begin{tabular}{|l|l|l|l|}
\hline $\begin{array}{l}\text { Parameter } \\
\text { Symbol }\end{array}$ & M/C 1 & M/C 2 & M/C 4 \\
\hline$K_{A}$ & 200 & 4 & 200 \\
$T_{A} \quad(\mathrm{~s})$ & 0.3575 & 0.02 & 0.02 \\
$T_{f}$ (s) & 1.0 & 0.05 & 1.0 \\
$K_{f}$ & 0.0529 & 0.05 & .01 \\
$E_{f \min }$ (p.u) & -5.73 & 0.0 & 0.0 \\
$E_{f \max }$ (p.u) & 5.73 & 4.46 & 7.32 \\
$G_{s}$ & 0.03 & 0.03 & 0.04 \\
\hline
\end{tabular}

Table 4: Parameters of transmission lines in p.u

\begin{tabular}{|l|l|l|l|}
\hline Bus \# & $R$ & $\mathrm{j} X$ & $\mathrm{j} Y$ \\
\hline $1-7$ & 0.0 & 0.12 & 0.0 \\
$7-8$ & 0.009 & 0.152 & 0.0688 \\
$8-9$ & 0.088 & 0.1055 & 0.0982 \\
$9-2$ & 0.0 & 0.12 & 0.0 \\
$9-6$ & 0.009 & 0.152 & 0.0688 \\
$6-10$ & 0.009 & 0.152 & 0.0688 \\
$10-3$ & 0.0 & 0.12 & 0.0 \\
$10-5$ & 0.0088 & 0.1055 & 0.0982 \\
$7-5$ & 0.009 & 0.152 & 0.0688 \\
$5-11$ & 0.009 & 0.152 & 0.0688 \\
$11-4$ & 0.0 & 0.12 & 0.0 \\
$11-12$ & 0.018 & 0.304 & 0.0344 \\
\hline
\end{tabular}

Table 5: Loads and operating points

\begin{tabular}{|l|l|l|}
\hline & $P+\mathrm{j} Q$ (p.u) \\
\cline { 2 - 3 } & Case \#1 & Case \# 2 \\
\hline Load 1 & $-0.5-\mathrm{j} 0.309$ & $-0.8-\mathrm{j} 0.48$ \\
Load 2 & $-0.3-\mathrm{j} 0.155$ & $-0.3-\mathrm{j} 0.18$ \\
Load 3 & $-0.25-\mathrm{j} 0.155$ & $-0.3-\mathrm{j} 0.18$ \\
Load 4 & $-0.25-\mathrm{j} 0.155$ & $-0.3-\mathrm{j} 0.18$ \\
M/C 1 & $0.12+\mathrm{j} 0.058$ & $0.2237+\mathrm{j} 0.14$ \\
M/C 2 & $0.2+\mathrm{j} 0.04$ & $0.5 \quad+\mathrm{j} 0.111$ \\
M/C 4 & $0.235+\mathrm{j} 0.154$ & $0.235+\mathrm{j} 0.2587$ \\
SCG & $0.75+\mathrm{j} 0.11$ & $0.75+\mathrm{j} 0.2037$ \\
\hline
\end{tabular}


Ragaey A. F. Saleh, "Particle Swarm-Aided Design of a Fuzzy Logic Stabilizer for a Superconducting...."
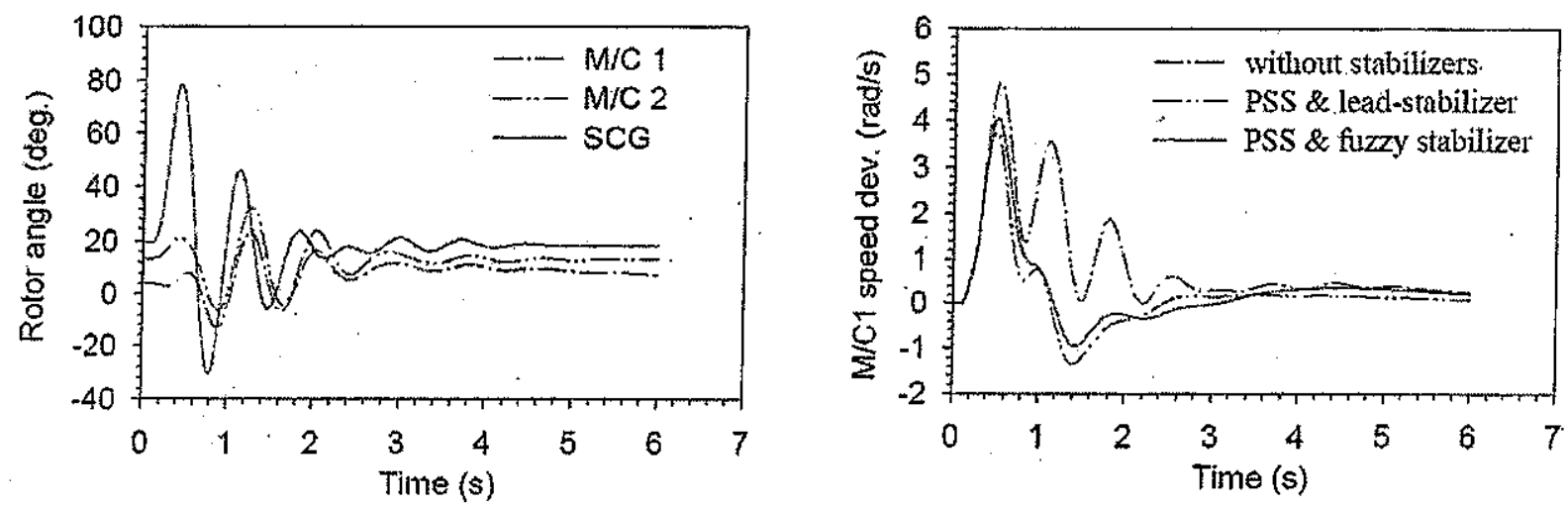

Fig.5(a) System response to $\mathrm{SC}$ at operating point \#1, all machines without stabilizers
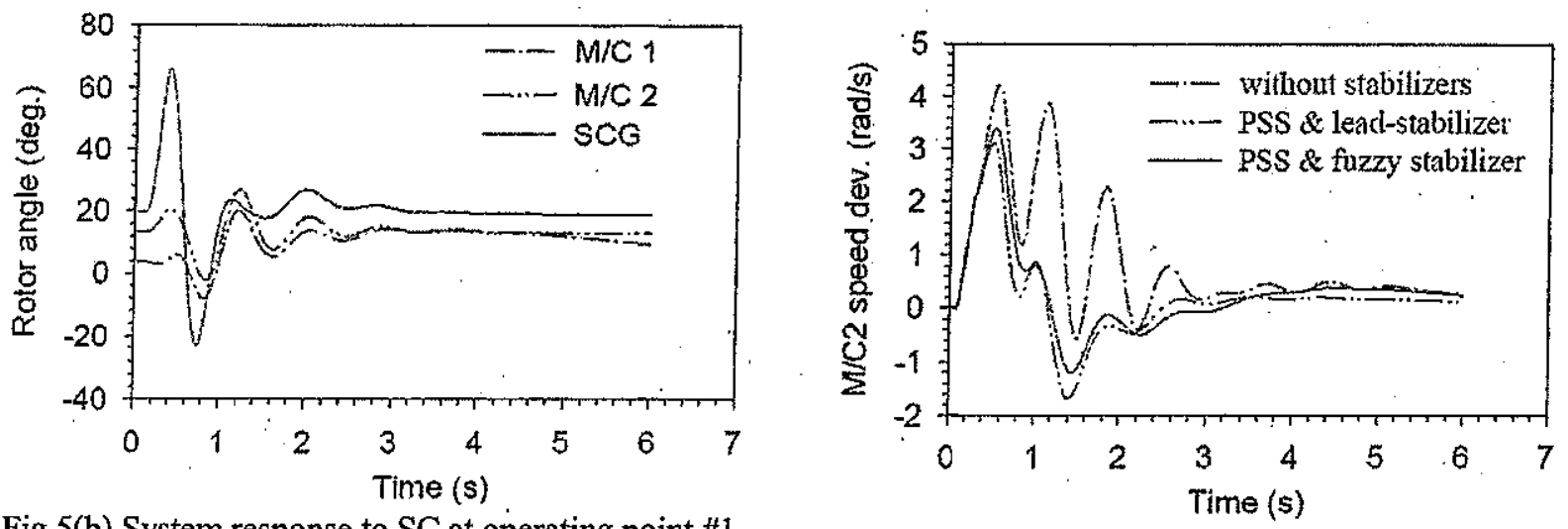

Fig.5(b) System response to $\mathrm{SC}$ at operating point \#1, conventional machines with PSS and SCG with leadstabilizer

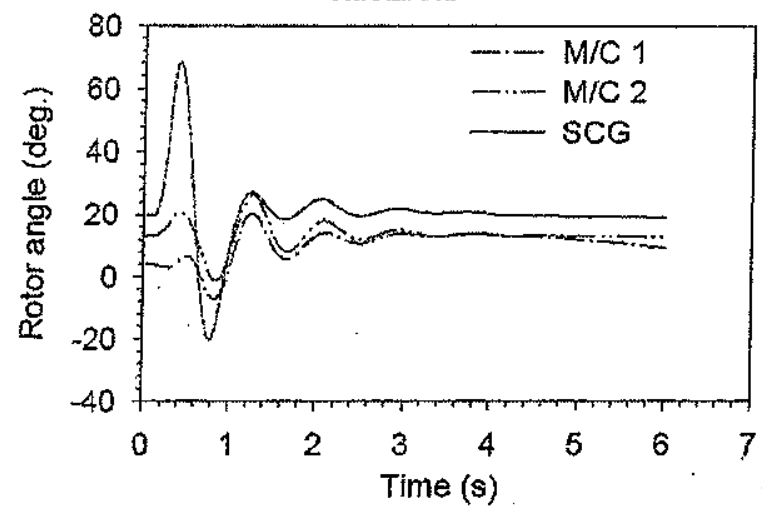

Fig.5(c) System response to $\mathrm{SC}$ at operating point \#1, conventional machines with PSS and SCG with fuzzy. logic stabilizer

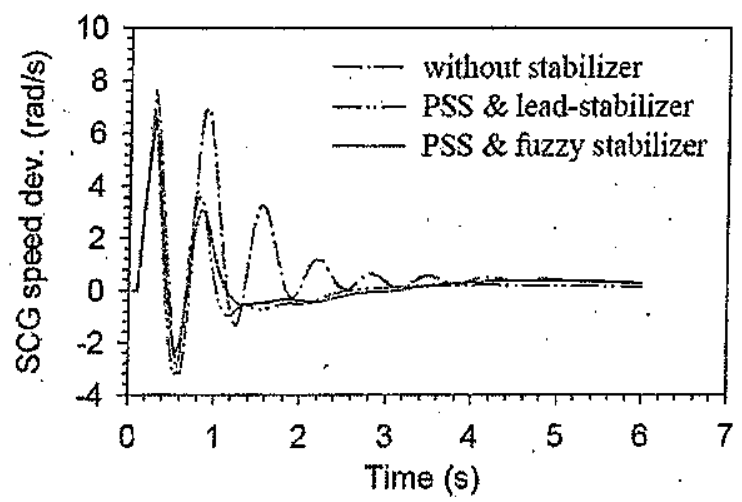

Fig.6. System response to $\mathrm{SC}$ for $200 \mathrm{~ms}$ at operating point \#1 
Ragaey A. F. Saleh, " Particle Swarm-Aided Design of a Fuzzy Logic Stabilizer for a Superconducting...."
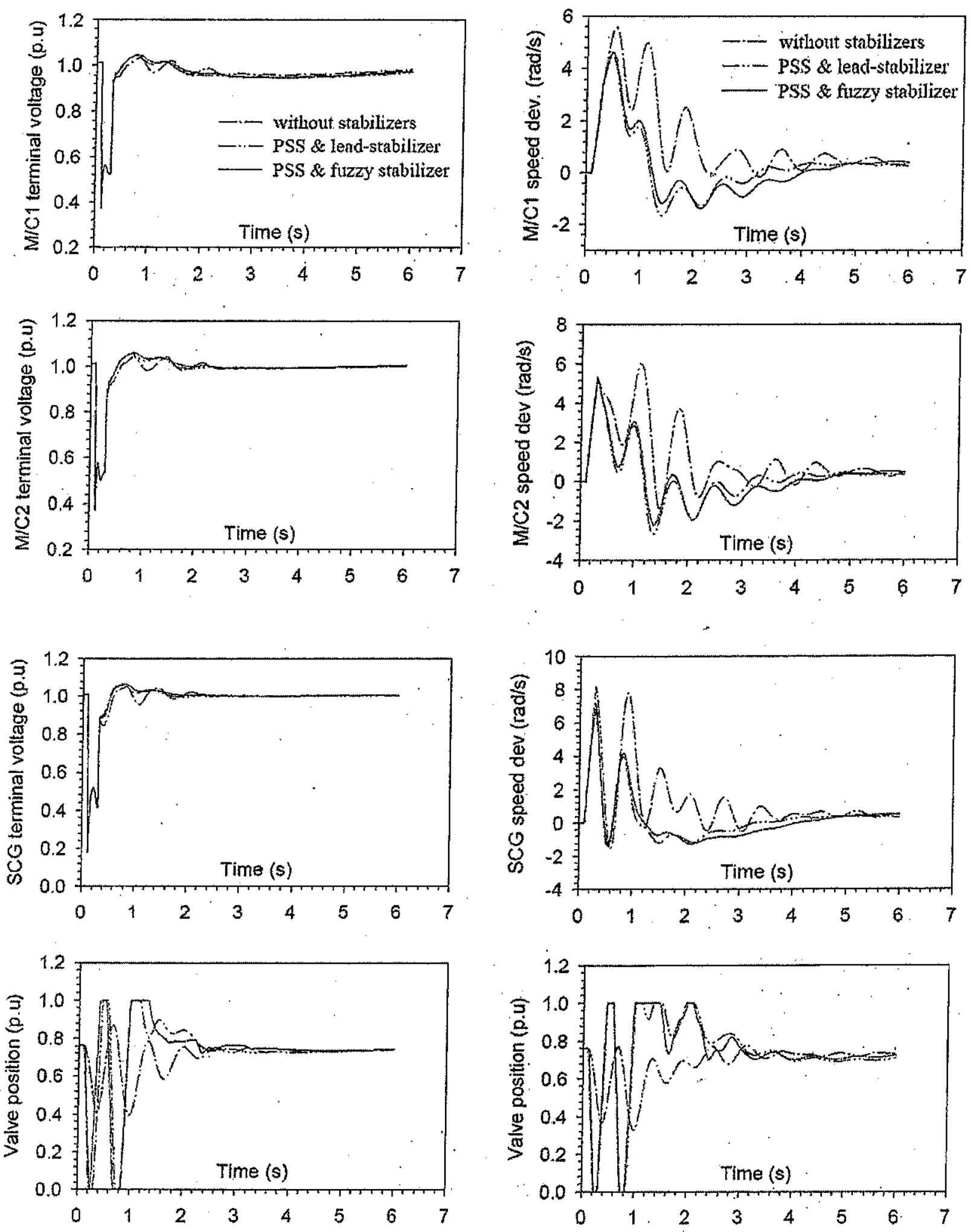

Fig.7 System response to SC for $200 \mathrm{~ms}$ at operating point \#1

Fig.8 System response to SC for $200 \mathrm{~ms}$ at operating point \#2 